\title{
Summary of the American College for Advancement in Medicine November 2005 Conference on Scientific Integrative Medicine: Advancing Health Horizons
}

\author{
Stephen A. Feig ${ }^{1}$, James Biddle ${ }^{2}$, Eleanor Hynote ${ }^{3}$, Neal Speight ${ }^{4}$, Kenneth Bock ${ }^{5}$, \\ Allan Magaziner ${ }^{6}$ and Joseph E. Rich ${ }^{7}$ \\ ${ }^{1}$ Integrative Medical Consulting, 208 Vista Bella Drive, Santa Cruz, CA 95060, ${ }^{2}$ Asheville Integrative medicine, \\ 832 Hendersonville Road, Asheville NC 28803, ${ }^{3}$ Phoenix Wellcare, 935 Trancas Street Suite 1A, Napa, \\ CA 94558, ${ }^{4}$ The Center For Wellness, 2317 Randolph Road, Charlotte, NC 28207, ${ }^{5}$ Albany Medical College, \\ Rhinebeck Health Center, 108 Montgomery Street, Rhinebeck, NY 12572, ${ }^{6}$ University of Medicine and \\ Dentistry of New Jersey, Robert Wood Johnson Medical School, 1907 Greentree Road, \\ Cherry Hill, NJ 08003 and ${ }^{7}$ The Center for Environmental \& Integrative Medicine, 9217 Parkwest Boulevard, \\ E-1 Knoxville, TN 37923, USA
}

The American College for Advancement in Medicine's (ACAM) 64th International Educational Symposium on Complementary, Alternative and Integrative Medicine (CAIM) was held on November 9-13, 2005 at the Anaheim Hilton Hotel in Anaheim, California. ACAM's Fall 2005 conference, 'Scientific Integrative Medicine: Advancing Health Horizons', offered a total of 70.5 AMA CME credits and brought together an international cadre of approximately 600 physicians, scientists and medical students/residents (from nine countries) for a fascinating educational program on the scientific and clinical evidence supporting the practice of a wide range of CAIM therapies.

Conference keynote presentations addressed a diverse breadth of common medical conditions and provided scientific substantiation for many of the most effective treatments used by physicians who have successfully incorporated CAIM into their practice of medicine. Presenters highlighted strategies for successfully using CAIM to address conditions such as hypothyroidism, hypertension, endothelial dysfunction, degenerative disc disease, osteoporosis, infectious diseases (bacterial and viral) and the autistic spectrum disorders. Keynote lectures also focused upon the effects of nutrientgene interactions and the vast spectrum of potential therapeutic interventions using nutrients to address and to compensate for

For reprints and all correspondence: Joseph E. Rich, Medical Director, The Center for Environmental \& Integrative Medicine, 9217 Parkwest Boulevard, E-1 Knoxville, TN 37923. Tel: (865) 694-9553;

Fax: (865) 694-7658; E-mail: mascots@pol.net common single nucleotide polymorphisms (SNPs). Presenters also emphasized the importance of epigenetic gene modification and they elucidated the mechanisms by which food, nutrients and environmental toxins, such as heavy metals, can modify gene expressions.

In a featured lunchtime awards ceremony, actress and author Suzanne Somers became the first recipient of ACAM's Legacy Award for her dedication to making the public more aware of the use of bio-identical hormones in the treatment of the periand post-menopausal symptoms. Her best selling book, The Sexy Years, featured interviews with many forward-thinking clinicians and noted integrative physicians who have helped bring bio-identical hormone replacement therapy into the forefront of the American public. During her moving presentation, Ms Somers emphasized the importance of physicians educating their patients with regard to treatment options that use bio-identical hormone replacement therapy. She commended medical organizations such as ACAM for their open-minded approach and for the training they impart to thousands of physicians and medical students. Ms Somers also highlighted her own innovative approaches and experience in her battle against breast cancer. The awards ceremony received rave reviews and $\mathrm{Ms}$ Somers received a standing ovation after her presentation. Dr Allan Magaziner, ACAM's outgoing president, proudly presented Ms Somers with ACAM's first Citizen's Legacy Award. ACAM's Citizens Legacy Award was established to recognize the outstanding contributions of non-physicians toward bringing greater public 
awareness to CAIM practices and to advancing CAIM therapies.

ACAM's incoming president, Kenneth Bock, MD, observed that the fall conference provided 'solid and scientifically based information supporting the field of integrative medicine and that the therapies, discussed by the presenters, were readily applicable to most physicians' practice of medicine'. Dr Bock assumed his leadership role as ACAM's president following the conference. During the conference, he emphasized ACAM's intention of 'building bridges with other CAIM organizations, as well as with more academic and conventional medical organizations.'

A complete list of the keynote conference presentations can be found following this article.

ACAM's Fall 2005 meeting also offered four in-depth 2-day pre-conference workshops along with a 1-day workshop on Scientific Writing. Two-day workshop themes included Prolotherapy, Hyperbaric Medicine, Nutrition and Metabolism, and Chelation and Heavy Metal Toxicity.

ACAM's Prolotherapy Workshop was Chaired by Jeffrey J. Patterson, DO, Professor of Family Medicine at the University of Wisconsin School of Medicine. He was assisted by Carl Osborn, DO and Jean Paul Ouellette, MD. Prolotherapy involves the injection of an irritant solution into the area where ligaments have either been weakened or damaged through injury or strain. The injection stimulates an inflammatory response that results in a tightening of aberrant ligamental laxity and produces biomechanical stabilization of the hyper-mobile region. The Prolotherapy Workshop consisted of lectures describing the general history of prolotherapy and the specific techniques employed in the practice of prolotherapy. Demonstrations of joint examinations and prolotherapy injections were accomplished with the use of patient models and video. This workshop allowed both the physician new to prolotherapy and the experienced prolotherapist to advance their skills and knowledge. Each of the two days of the workshop concluded with a panel discussion that reviewed case histories, prolotherapy 'pearls', and common complications encountered in the practice of prolotherapy. Dr Patterson commented, 'It was exciting to work with ACAM on this workshop. We found the people in the organization to be stimulating and eager to explore prolotherapy as a treatment for chronic [musculoskeletal] pain.'

Dileep S. Sachan, DVM, PhD, Professor in the Department of Nutrition at the University of Tennessee and a board member for the American College of Nutrition, chaired ACAM's Nutrition and Metabolism Review Workshop. The workshop reviewed the fundamentals of physiology and biochemistry as applied to the metabolism of various macro- and micronutrients, including those involved with a number of inborn errors of metabolism. Aspects of drug metabolism, nutrient-drug interactions and food allergy were also discussed. The pathophysiologies of acute and chronic disease conditions were reviewed and nutritional interventions in these conditions were addressed. The workshop's teaching faculty reviewed some of the nutritional requirements inherent in conditions such as metabolic syndrome, lipid dystrophies and disorders of the gastrointestinal tract, and the hepatobiliary, renal and cardiovascular systems. They also reviewed nutritional requirements through the life cycle as well as nutritional status assessment and methods of feeding.

ACAM's Hyperbaric Oxygen Therapy (HBOT) Workshop was moderated by Joseph Rich, MD MPH, Director of the Center for Environmental and Integrative Medicine in Knoxville, Tennessee. HBOT is the periodic treatment of the entire body with $100 \%$ oxygen at greater than normal atmospheric pressures. The workshop convened a distinguished international group of physicians and scientists who addressed the medical uses of HBOT in a diverse range of disorders, including autism, cerebral palsy, stroke/brain injury, non-healing wounds, osteomyelitis and Lyme disease. Dr Rich commented, 'Hyperbaric oxygen therapy is an underutilized safe and effective therapy that has a surprisingly broad spectrum of application for a wide number of medical disorders. I urge progressive minded physicians to become more familiar with HBOT and not to hesitate in employing it in many acute and chronic diseases.' The HBOT Workshop allowed attendees to gain a broad understanding of the principles and practical applications of HBOT in medicine.

Edwin Cooper, PhD, Distinguished Professor, Department of Neuroimmunology, at the David Geffen School of Medicine at University of California, Los Angeles, chaired ACAM's Writer's Workshop. The workshop helped participants interested in developing their scientific writing skills to develop a foundational perspective in the art and science of writing for the peer-reviewed scientific literature. Several participants brought their work for review and received constructive advice on optimizing their presentations for submission to medical journals.

Overall, conference attendees were very impressed with the scientific and clinical evidence, which was presented in support of CAIM therapies. Dr Bock commented 'as the incoming president of ACAM, it is a thrill for me to be a part of such a positive event, and I greatly look forward to our next meeting in Dallas, Infection and Chronic Illness: Integrative Approaches to Lyme Disease, Stealth Infections and Inflammation, which looks to be incredibly thought provoking and very practical for any practitioners involved in the treatment of chronic illness.

Anyone who missed the opportunity to attend ACAM's scientific conference 'Scientific Integrative Medicine: Advancing Health Horizons' or the pre-conference workshops on 'Scientific Writing', 'Prolotherapy', 'Hyperbaric Medicine', 'Nutrition and Metabolism', and/or 'Chelation and Heavy Metal Toxicity' can order DVDs/CDs from http://www.acam. org or through telephone (800-532-3688 or 949-583-7666).

The American College for Advancement in Medicine (ACAM) is a not-for-profit professional medical organization for physicians interested in learning about evidence-based CAIM therapies. ACAM's goals are to improve skills, knowledge and diagnostic procedures as they relate to 
complementary and alternative medicine; to support research and to develop awareness of alternative methods of medical treatment. ACAM is dedicated to educating physicians and other health care professionals on the latest findings and emerging procedures in CAIM, botanical medicine, environmental medicine and in the use of effective evidence-based nutritional therapeutics. As the voice of CAIM, ACAM strives to increase awareness and recognition of complementary, alternative and integrative medical therapies, and play an active role in shaping the future of the CAIM movement.

ACAM represents nearly 1000 physicians in 30 countries and is the largest and oldest organization of its kind in the world dedicated to providing its members with educational excellence in integrative medicine. ACAM's leading edge educational programming facilitates the incorporation of clinically relevant biomedical therapies by fast-tracking scientific advancements in nutritional medicine, nutraceuticals and ethnopharmacology into the practice of cutting edge CAIM medicine.

ACAM was the first major medical organization to educate its members about such now well-accepted concepts as insulin resistance/metabolic syndrome, the detrimental effects of homocysteine, the protective effects of antioxidants, the clinical uses of essential fatty acids, cancer chemoprotection therapies with diet and herbs, testing for cardiosensitive C-reactive protein, the benefits of natural thyroid replacement and nutritional medicine as applied to psychiatry.

ACAM's next scientific conference 'Infection and Chronic Illness: Integrative Approaches to Lyme Disease, Stealth Infections and Inflammation' (approved for 17 AMA ACCME hours) will be held at the Intercontinental Hotel in Dallas Texas, from May 3-7, 2006. Pre-conference workshop topics include 2-day trainings (approved for 15 AMA ACCME's) in 'Metal Toxicology', 'Oriental Medicine' and 'Hyperbaric Oxygen Therapy'. ACAM will introduce two exciting new 1-day workshops in Dallas, IV Therapies In Clinical Practice', and 'Integrating CAIM into Clinical Practice', which are both approved for 7.5 AMA ACCME credits.

For further information, please see http://acam.org/ DALLASabout.html or contact ACAM at +1-800-532-3688 (in the USA) or +1-949-583-7666 (outside the USA).

\section{Summary of Presentations}

\section{David Brownstein, MD}

Dr Brownstein is a Clinical Assistant Professor of Family Medicine at Wayne State University and author of seven books.

\section{The Clinical Use of Iodine}

Dr Brownstein reviewed the history of iodine including aspects about its discovery, the recommended daily allowances, and its widespread deficiency in the modern diet. He highlighted studies suggesting maternal imbalance of iodine may be related to impaired psychoneurological development in their children. He also noted that areas with the highest incidence of goiter have higher rates of sudden infant death syndrome. Dr Brownstein went on to discuss the physiology of iodine and conditions successfully treated with iodine therapy. He highlighted attention deficit disorder, fibrocystic breast disease, thyroid disorders, ovarian cysts, peyronie's disease, infections, keloids and others. Dr Brownstein emphasized that the breast is the second major storage compartment for iodine just behind the thyroid and that diseased breast tissue, such as that found in fibrocystic disease, will likely require more iodine because of greater tissue depletion.

\section{Mark Houston MD}

Dr Houston is a Clinical Professor of Medicine at Vanderbilt University School of Medicine and Chief of the Nutrition Division at Saint Thomas Medical Group, Saint Thomas Hospital in Nashville, Tennessee.

\section{The Natural Treatment of Hypertension}

Dr Houston reviewed the role of vascular biology in the pathogenesis of hypertension. He discussed the environmental and genetic interactions with micronutrients and their role in modulating vascular inflammation in a manner that may result in potentially less hypertension and end organ damage. Dr Houston reviewed the wealth of scientific evidence that confirms that oxidative stress, inflammation and immunologic dysfunction initiate and propagate hypertension and cardiovascular disease. He also summarized the mechanism of action for the role of numerous nutrients, including amino acids, minerals bioflavonoids and fatty acids, in reversing hypertension.

\section{Konrad Kail, ND}

Dr Kail the co-founder of the Southwest College of Naturopathic Medicine and Health Sciences and a recent advisor to the National Center for Complementary and Alternative Medicine at the NIH.

\section{Managing Subclinical Hypothyroid}

Dr Kail reviewed the risks associated with the syndrome of Subclinical Hypothyroidism introducing a new management paradigm that optimizes thyroid function based on resting Metabolic Rate and Brachioradialis Reflexometry. He reviewed factors affecting thyroid function including lead, mercury, PCBs, fungicides and medications and highlighted nutrients that help restore its function. Additionally, he made the case for potentially significant declines in health based on untreated subclinical hypothyroidism, including increased cardiovascular, neurological and endocrinological complications. 


\section{Thomas Levy, MD, JD}

Dr Levy was formerly an Assistant Professor of Medicine at Tulane Medical School and is currently a Fellow of the American College of Cardiology, and the author of four books.

\section{Vitamin C: Past, Present and Future Applications}

Dr Levy reviewed the strong role of Vitamin $\mathrm{C}$ in the prevention of infection, treatment of scurvy and neutralization of toxins. He made the very important point that all infections and toxins will induce a localized and/or systemic Vitamin C deficiency, which in its extreme form, may induce injury to lipids. He reviewed the documented record of success of Vitamin $\mathrm{C}$ in treating encephalitis, mononucleosis, diphtheria, viral pneumonia, tetanus, mushroom poisoning, snakebites and even barbiturate overdose. He reviewed vitamin C's role in dental disease and the unparalleled track record of safety of this key nutrient.

\section{Joseph C. Maroon, MD}

Dr Maroon, the Vice Chairman of the Department of Neurological Surgery at the University of Pittsburgh School of Medicine and the team neurosurgeon for the Pittsburgh Steelers.

\section{Omega 3 EFA for Spine Pain}

Dr Maroon reviewed the role of Omega 3 essential fatty acids in the treatment of inflammation and highlighted the results of his study using Omega 3 fatty acids in the treatment of 250 non-surgical neck or back pain patients. At the end of the study, $60 \%$ of the participants stated their overall pain was improved and $60 \%$ stated specifically that their joint pain had improved. Overall, 80\% stated they were satisfied with their improvement and $88 \%$ stated they would continue to take fish oils. Dr Maroon reviewed other botanical therapies for inflammation including Green Tea, Turmeric, White Willow Bark, Ginger and Boswellia. He also addressed the dosages of Omega 3 oils necessary to control pain and he contrasted the negligible side effects of Omega 3 oils with the welldocumented adverse drug reactions from over the counter and prescription non-steroidal anti-inflammatory medications.

\section{Amy Yasko, ND, PhD}

Amy Yasko, ND, PhD, received her doctorate from Colgate University in Microbiology, Immunology and Infectious Disease and studied Naturopathy at Clayton College of Natural Health.

\section{Nutrigenomics, Methylation and RNA-Based Nutrients}

Dr Yasko discussed the laboratory studies that allow for testing for genetic susceptibility to disease and she extensively discussed the science of nutrigenomics. 'Nutrigenomics' is defined as the integration of concepts in molecular biology and genomics in order to study the ability of foods and nutritional supplements to interact with genes and to influence our health and lower the genetic risk component for multifactorial disease. Dr Yasko described the methionine/folate pathway as a central pathway that is especially amenable to nutrigenomic screening. Defects in methylation can make one more susceptible to diabetes, cardiovascular disease, thyroid dysfunction, neurological inflammation, neurotransmitter imbalances, atherosclerosis, cancer and autism. Dr Yasko also described how defects in methylation can make one more susceptible to the toxic effects of heavy metals, especially arsenic and mercury. Dr Yasko described how heavy metals can decrease the brain neurotransmitters, such as serotonin and dopamine, if there are defects with $\mathrm{BH} 4$ and $\mathrm{BH} 2$ genes. By identifying the precise areas of genetic fragility, it is possible to target appropriate nutritional supplements. Dr Yasko discussed the potential uses of specific RNA-based supplementation.

\section{John Longhurst, MD, PhD}

John Longhurst, MD, PhD, earned both his Medical Degree and his $\mathrm{PhD}$ in Cardiovascular-Pulmonary Physiology from the University of California at Davis. He is now Associate Dean at UC Irvine and currently oversees the Susan Samueli Center for Complementary and Alternative Medicine.

\section{The Role of Acupuncture in Modern Healthcare, Mechanistic Focus on the Cardiovascular System}

Acupuncture is a 3000-year-old treatment and is known to be useful in a number of chronic conditions such as pain, depression, recurrent nausea and vomiting among other conditions. Only recently has acupuncture been implicated in the treatment of cardiovascular diseases. Dr Longhurst described acupuncture's usefulness in the treatment of hypertension and chronic chest pain associated with myocardial ischemia. He presented his laboratory's research on the cardiovascular influence of electroacupuncture (EA). His laboratory has shown that EA at the Jianshi-Neiguan acupuncture points stimulates the underlying median nerve to provide sensory information through afferent nerve fibers to the central nervous system, particularly to specific nuclei in the hypothalamus and midbrain that are concerned with the regulation of the sympathetic nervous system. Dr Longhurst described how manual acupuncture and EA can also cause similar sympathetic response to the cardiovascular system and reduce blood pressure and ameliorate cardiac dysfunction in an experimental model of demand induced myocardial ischemia. It appears that one of the ways that acupuncture has clinically significant effects is through a number of opioid and opiod-like modulatory neuropeptides that reduce the activity of neurons in the brain.

\section{Leo Galland, MD}

Leo Galland, MD author of 'Super Immunity for Kids' and 'Power Healing'. 


\section{Interactions in Clinical Practice: Drug-Supplement, Drug-Nutrient}

Dr Galland explained that over half of all prescription medication have the ability to deplete specific nutrients. Dietary supplements can interact with medication affecting absorption and toxicity of many drugs. Coenzyme Q10, folic acid, magnesium, zinc, vitamins B2 and B6 are the nutrients most commonly depleted. Mechanisms by which drugs influence nutrients include the impairing of absorption or bioactivation and through increasing excretion. Examples of clinically significant nutrient depletions include statin and Adriamycin depletion of Coenzyme Q10, Cisplatin depletion of magnesium, Thiazides and 5-ASA derivatives depletion of folate, and loop diuretics depletion of a wide range of nutrients, including potassium, magnesium, calcium, certain B-vitamins and Vitamin C. Dr Galland described some beneficial drug-nutrient interactions such as the protective effects on acetaminophen toxicity from the use of N-acetylcysteine, L-methionine, SAMe, milk thistle and schisandra. Protective supplements for aspirin and NSAID gastropathy (in human trials) include Vitamin C, SAMe, deglycyrrhizinated licorice and colostrum.

\section{Stephen Sinatra, MD}

Stephen Sinatra, MD is a Board Certified Cardiologist and fellow of the American College of Cardiology and the American College of Nutrition.

\section{Metabolic Cardiology: A New Paradigm for The Prevention and Treatment of Heart Disease}

Dr Sinatra's presentation introduced the concept of 'metabolic cardiology' and highlighted the use of metabolic therapies in heart disease. Metabolic therapy involves the administration of a substance, that is normally found in the body, to enhance a metabolic reaction within the cell. Dr Sinatra extensively discussed the biology of Coenzyme Q 10, a lipid soluble antioxidant that plays vital roles in cellular ATP processes and thereby influences both systolic and diastolic function. Dr Sinatra suggested that mitochondrial dysfunction may result in symptoms of heart disease through some association with Coenzyme Q 10 levels. As studies indicate that women cannot make as much Coenzyme Q 10 as men and that women with mitral valve prolapse and hypertension have more diastolic dysfunction, since diastole requires more cellular energy than systole and is more dependent on ATP, Dr Sinatra hypothesized that women maybe more susceptible to diastolic dysfunction due to lower Coenzyme Q10 and resultant lower ATP. Dr Sinatra also discussed nutraceuticals that support cardiac metabolism, such as D-Ribose and L-Carnitine. D-Ribose is a naturally occurring pentose sugar that rebuilds the energy stores in the cell. In the myocardium, the rate-limiting step in the salvage and synthesis of ATP is the availability of D-Ribose. These three compounds,
Coenzyme Q10, L-Carnitine and D- Ribose formed the triad of Sinatra's Metabolic Cardiology.

\section{Randolph M Howes, PhD, MD}

Randolph M Howes, $\mathrm{PhD}$, MD completed residency in general surgery and plastic surgery while doing basic research in oxygen-free radicals at Johns Hopkins University. He invented the triple lumen catheter and, in 2004, published the first selective world review on oxygen metabolism in his book: UTOPIA (Unified Theory of Oxygen Participation in Aerobiosis).

\section{Pro-oxidant Protection and Oxidative Self-Healing}

Dr Howes discussed his research in free radical biology and stated that his belief the free radical theory is unfounded and that electronically modified oxygen derivatives (EMOD) are of low toxicity and are essential for energy production, pathogen protection, secondary messenger signaling and as tumoricidal agents. His unified theory states that EMOD deficiency levels allow for the manifestation of diseases, including neoplasia, and is integral in the aging phenomena. Dr Howes described how antioxidants become pro-oxidants. He summarized his presentation by discussing how antioxidants have failed to control aging and disease and he stated that the scientific literature is increasingly showing that antioxidants can harm biological systems.

\section{Michael Uzick, ND}

Dr Uzick received his doctorate of Naturopathic Medicine from Bastyr University in Seattle Washington. He practices in Tucson Arizona and lectures on alternative therapies for cancer, hepatitis $\mathrm{C}$ and environmental toxicities.

\section{Natural Strontium: A Highly-Effective Treatment for Osteoporosis}

Dr Uzick reviewed the fundamentals of osteoporosis and introduced evidence that strontium can be a safe and an effective adjunctive osteoporosis therapy. He pointed out that conventional drug therapies using bisphosphonates are associated with esophageal complications, inflammatory conditions and osteonecrosis of the jaw. Natural treatments that include calcium and other minerals, vitamin $\mathrm{D}$ and vitamin $\mathrm{K}$ are both safe and effective. Strontium is a non-toxic natural mineral that was first reported in 1959 by the Mayo Clinic to be useful for improving pain in severe osteoporosis. More recently, several studies have documented the benefits of strontium for both improving Dual Energy X-ray Absorptiometry (DEXA) scores and in dramatically reducing fracture rates. When supplementing with strontium and calculating bone mineral density (BMD), DEXA scores must be adjusted to produce a 'strontium-adjusted BMD' due to strontium's blockage of X-rays. At $2 \mathrm{~g}$ of strontium per day, studies have shown as much as a $49 \%$ decreases in fracture risk by one year, a $14 \%$ increase in lumbar BMD and 12\% decrease in C-telopeptide. 
Bone biopsies from hundreds of animals and humans, who have consumed strontium, show normal mineralization and no cellular toxicity. Side effects were no greater than placebo in most studies.

\section{Jack Challem}

Jack Challem is author of Feed Your Genes Right, The Inflammation Syndrome, and Syndrome X. He writes and publishes The Nutrition Reporter ${ }^{T M}$ newsletter. He has also published original scientific articles in several journals, where he has focused on the role of free radicals and antioxidants in primate evolution.

\section{Feed Your Genes Right: A Conceptual Framework for Nutrient-Gene Interactions}

Mr Challem described the biology of nutritional cofactors, which affect gene structure and function, as well as the role of genetic variations, which induce unique nutrient needs. Using vitamin B-1 as an example, he pointed out that deficiency in lab rats turns off 1000 genes and turns on 664 other genes. Similar effects are likely for most nutritional deficiencies. Mr Challem pointed out that the vast majority of people in our population have many nutrient deficiencies. Mr Challem made a case that the future of genetics research lies in the field of 'Epigenetics', which is the science devoted to the modifiers of gene function and expression, rather than just looking at effects of gene structure. He described how methylation often silences genes while acetylation usually expresses them. $\mathrm{Mr}$ Challem discussed how epigenetics can even be used to explain multi-generational observations, since the effects of nutrition and exercise have been observed to affect the health of future generations.

\section{Laura Power, PhD}

Dr Power is a nutritional biochemist, with a Masters degree in Nutritional Sciences from the University of Maryland and a Doctorate in Holistic Clinical Nutrition from International University. Her area of expertise is in the fields of immunology, allergy and epidemiology.

\section{The 5 Biotypes Diets}

Dr Power presented her 20 years of unique research correlating blood types and ancestry to food allergies. Her research identifies five diets based on ABO blood types (A1, A2, B, O and $\mathrm{AB}$ ), plus three subtypes based on being Rh-negative and one's gender. These blood types were correlated statistically to four kinds of food allergies-IgE, IgG, Lectins and T-cell mediated. She summarizes these results in her Diet Compass $^{\mathrm{TM}}$, a diagram that denotes the evolutionary origin of the different dietary strategies.

\section{Efrain Olszewer, MD}

Dr Olszewer is the Director and Professor of the Brazilian Foundation of Advanced Health Sciences, which teaches postgraduate courses in medicine. He is the author of 38 books and dozens of research articles.

\section{EDTA, Calcium, Sodium and Suppositories as a Therapeutic Approach for Chronic Degenerative Vascular Diseases}

Dr Olszewer discussed the administration of EDTA for vascular disease and the effectiveness of different forms of EDTA and different routes of administration. He began by stating that, many years ago, his group in Brazil switched from the 'standard bottle' of using $3 \mathrm{~g}$ of EDTA to the '1/2-bottle' of using $1.5 \mathrm{~g}$ of EDTA, because they found it just as effective in prior studies. He presented data from a study of 12 patients with stable angina where he compared the effectiveness of IV Mg-NaEDTA with rapid IV CaEDTA and NaEDTA rectal suppository. The IV treatments were administered once weekly and the suppositories were five days per week. The total weekly dose of EDTA was $1.5 \mathrm{~g}$ in each arm, administered over $90 \mathrm{~min}$ for the MgNaEDTA, administered over 15 minutes for the CaEDTA, and administered over a period of five days for each of the $300 \mathrm{mg}$ EDTA suppositories. The study went for 12-15 months, and IV patients received a total of 25 infusions. The outcomes measured were chest pain on a stress test. Positive results were observed in $87 \%$ of the MgNaEDTA patients, $69 \%$ of the CaEDTA patients and $53 \%$ for those who used suppositories. After 20 weeks, non-responders were moved from the CAEDTA and suppository arms to the MgNaEDTA arm, where they also reached an $87.5 \%$ positive response rate.

A second study looked at peripheral vascular disease in 24 patients, with positive improvements noted in $93 \%$ for the MgNaEDTA group, $33 \%$ for CaEDTA group and $17 \%$ for suppository group. Again, when non-responders were moved to the $\mathrm{MgNaEDTA}$ arm, they achieved a positive response rate of $83 \%$.

A third study looking a strokes at less than 6 months, with 14 patients showed a similar pattern in clinical improvement of $87.5 \%$ for MgNaEDTA, $66.6 \%$ for CaEDTA and $33.3 \%$ for suppositories. 12.

Three patients were moved to the group receiving MgNaEDTA and all three improved. A fourth study, analyzing lead toxicity in the hair of 14 patients, showed positive responses in all patients in all three arms. There was a slight trend for patients in the CaEDTA arm to have rising creatinine levels, which decreased after changing them to the MgNaEDTA arm. Dr Olszewer's concluded that IV MgNaEDTA is the best approach for vascular disease, and the IV CaEDTA is not as effective, but it can be used for patients who will not sit still for the 90 min IVs. All three approaches are powerful tools for lead detoxification. The NaEDTA suppositories are effective only for lead intoxication, not for vascular disease. 


\section{Alan Miller, ND}

Dr Miller is a 1989 graduate of Bastyr University. He is the senior technical advisor for Thorne Research and is the senior editor of Alternative Medicine Review, the MEDLINE-indexed journal published by Thorne Research. He has authored over 20 scientific papers.

\section{L-Arginine and its Effects On Endothelial Function and Cardiovascular Health and Disease}

Dr Miller reviewed endothelial function and pathophysiology and then discussed the roles of nitric oxide (NO) and L-arginine on blood vessel dilation. L-arginine is made primarily in the kidneys from L-ornithine and L-citrulline, with 3-5 g derived from dietary protein sources. Dr Miller noted that many studies have shown improved endothelial function after giving L-arginine. Nitric Oxide Synthase (NOS) activity is inhibited by ADMA (asymmetric dimethylarginine), which is increase in hyperlipidemia, hypertension, diabetes, $\mathrm{CHF}$ and renal failure. Supplemental L-arginine can reverse the detriments of ADMA by restoring endothelial NOS activity. Larginine has been shown to improve the vascular dilation effect of statin drugs, improve exercise duration in $\mathrm{CHF}$, reduce homocysteine, lower blood pressure and increase cardiac perfusion by $22 \%$. Dr Miller noted that L-arginine works in complement with nutraceuticals such as B-vitamins, fish oil, red rice yeast extract and CoQ-10.

The authors wish to thank Michael Janson MD for his proofreading and editing of this article. 


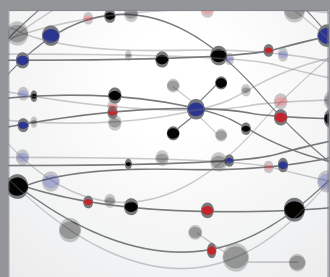

The Scientific World Journal
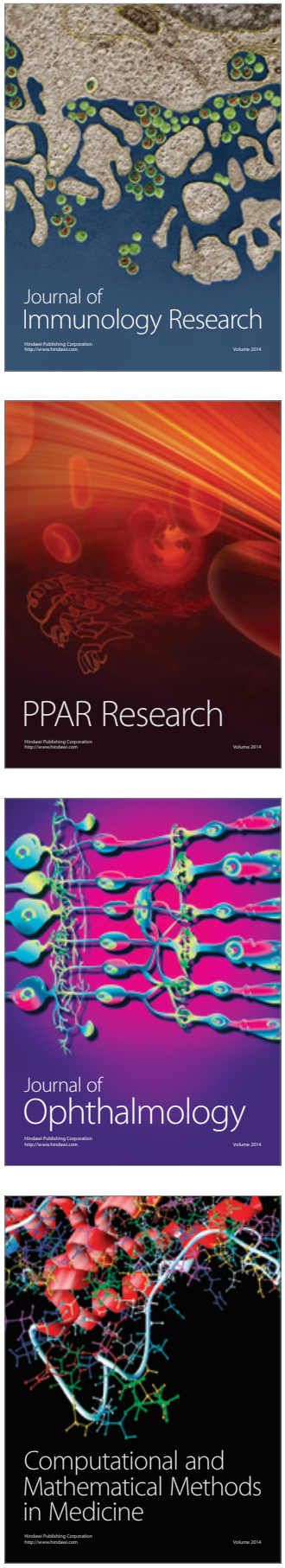

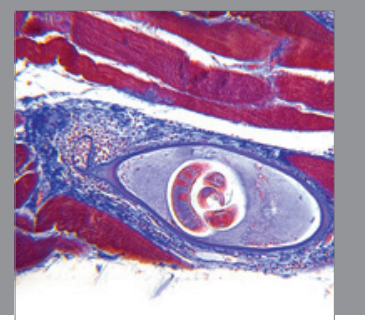

Gastroenterology

Research and Practice


\section{Hindawi}

Submit your manuscripts at

http://www.hindawi.com
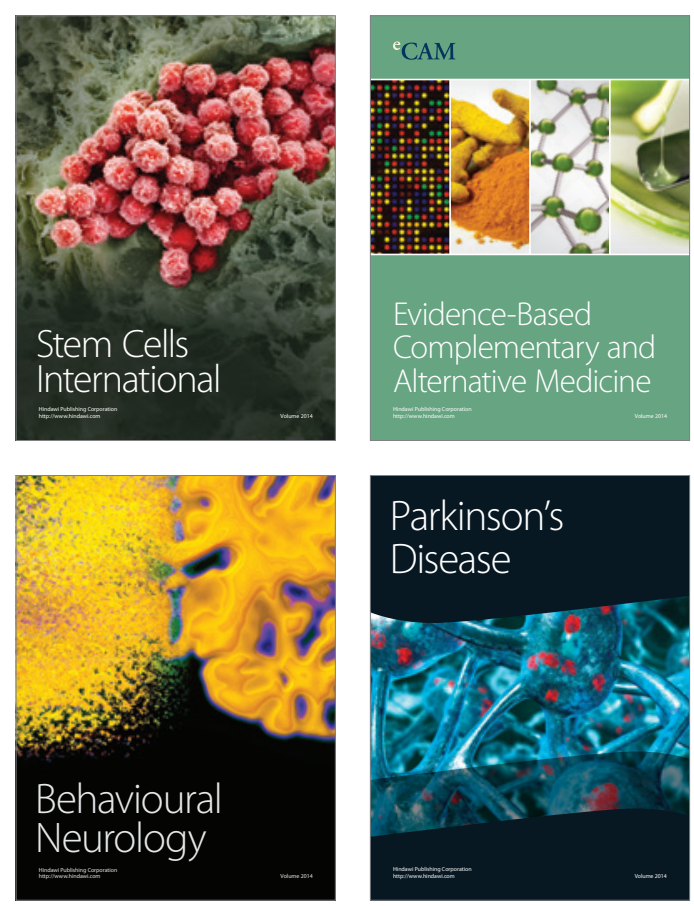

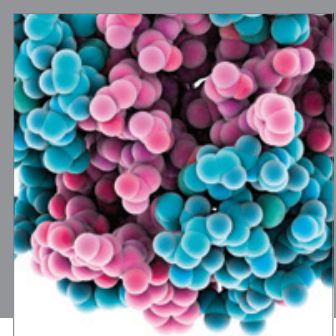

Journal of
Diabetes Research

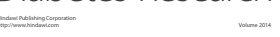

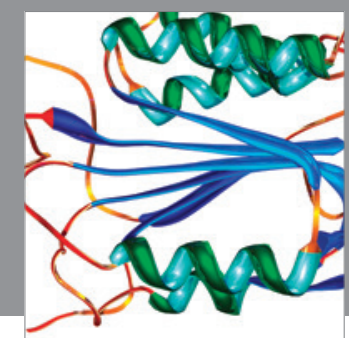

Disease Markers
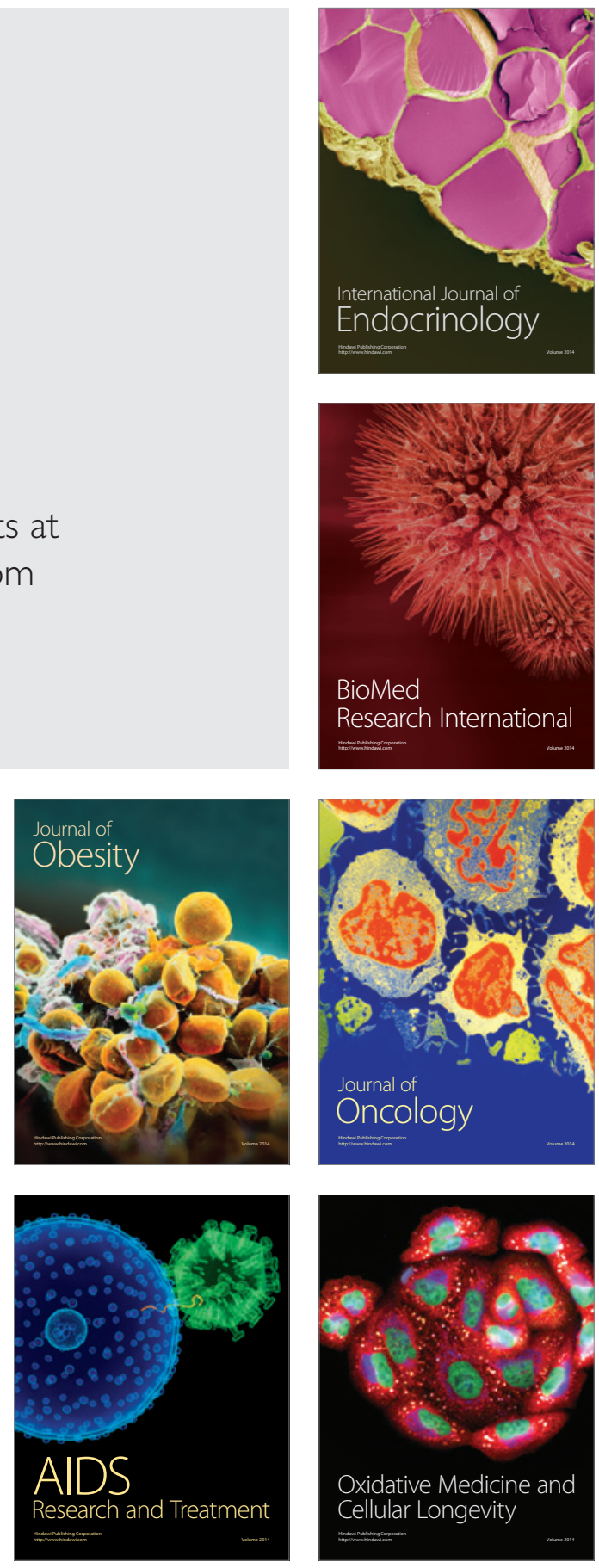